\title{
Rectosigmoid Endometriosis Causing an Acute Large Bowel Obstruction: A Report of a Case and a Review of the Literature
}

\author{
Gupta RK 1 ,Agrawal CS', Yadav RP', Uprety $D^{2}$, Sah $\mathrm{PL}^{3}$ \\ 'Department of Surgery, ${ }^{2}$ Department of Obstetrics and Gynecology, ${ }^{3}$ Department of Radiology, B.P. Koirala Institute of \\ Health Sciences, Dharan, Nepal
}

\begin{abstract}
Endometriosis is often seen in gynecology practice and is treated medically. However, intestinal involvement of endometriosis causing acute large bowel obstruction is uncommon and is difficult to differentiate from malignancy before surgery, owing to its similar colonoscopic and radiologic findings. We report a successfully-treated case of a 30-year-old woman in which endometrial infiltration of the large bowel caused acute obstruction, requiring emergency surgery to relieve the symptom and confirm the diagnosis. We present this unusual disease in general surgical practice and also review the literature.
\end{abstract}

Keywords: colorectal malignancy, endometriosis, intestinal obstruction

\section{INTRODUCTION}

Endometriosis is the presence of functional endometrial tissue consisting of glands and/or stroma located outside the uterus occurring in $5 \%-15 \%$ of menstruating women ${ }^{1}$.It affects the intestinal tract in $3-37 \%$ of all patients with pelvic endometriosis ${ }^{2}$. The differential diagnosis of colorectal endometriosis from malignancies of the colon and rectum is difficult due to similar colonoscopic and radiologic findings. Endometriosis causing acute bowel obstruction is relatively rare $^{3,4}$.

We present this unusual disease in general surgical practice because of its rarity and also review the literature.

\section{CASE REPORT}

A 30-year-old nulliparous woman presented to the emergency room with complaints of pain abdomen of 6 days duration, feculent vomiting, progressive abdominal distension and absolute constipation. She gave a history of altered bowel habits for the last one year characterised by passage of loose stool 3 - 4 times per day followed by constipation on the next day that was unrelated to menstruation. Oral laxatives improved the condition but the constipation recurred when she was without medication. She also gave a history of dysmenorrhea and dyspareunia. On physical examination, she was lethargic, dehydrated
Correspondence:

Dr. Rakesh Kumar Gupta

Department of Surgery

BP Koirala Institute of Health Sciences

Dharan, Nepal.

Phone: 00977-9842040265

Email: rakesh154@yahoo.co.in 
and had tachycardia. Abdominal examination revealed generalized distension and palpable bowel loops. Liver dullness was not obliterated and bowel sounds were exaggerated. Rectal examination revealed ballooning of the rectum and the non-tender uterus was felt anteriorly, and it was hard in consistency. An abdominal radiograph revealed the grossly dilatated large and small bowel with mucosal edema (Figure 1).

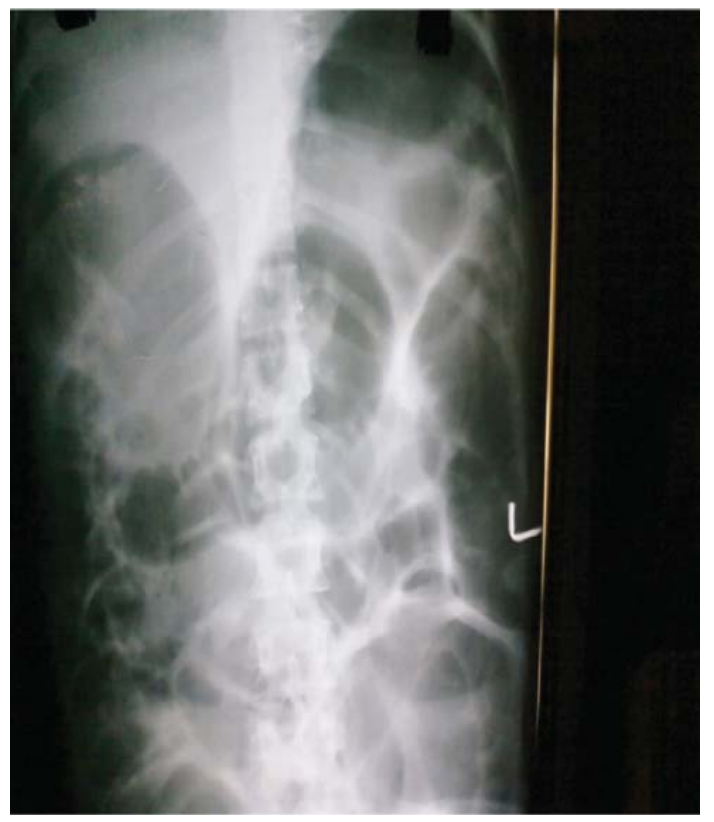

Figure 1: Abdominal radiograph showing a gross dilatation of the large and small bowel with mucosal edema

Flexible sigmoidoscopy revealed irregular mucosal swelling with a stricture $10 \mathrm{~cm}$ above the anal verge, which impededed the further advancement of the scope. There was no active bleeding at the time of the examination. Abdominal computed tomography (CT) showed a suspicious lesion in the recto-sigmoid junction of the colon with regional enlarged lymph nodes and ascites. The patient was taken up for emergency laparotomy after resuscitation with a provisional diagnosis of acute large bowel obstruction. At surgery, severe fibrosis over the Douglas pouch and pelvic cavity was noted. A chocolate cyst of $3 \times 3 \mathrm{~cm}$ was present in each of the ovaries with an intraperitoneal rupture of the left ovarian cyst and a grade IV endometriosis (Figure 2).

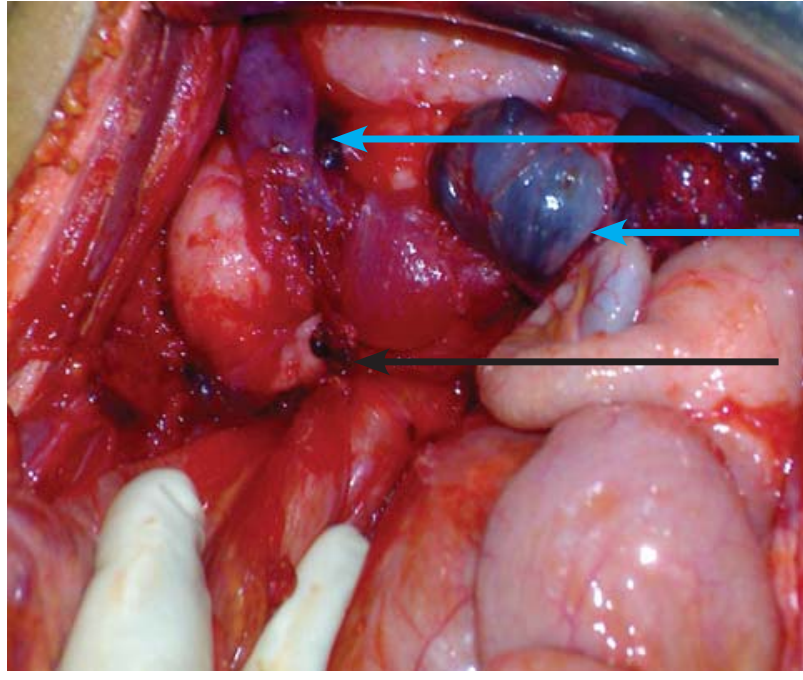

Figure 2: Operative photograph showing severe fibrosis over the Douglas pouch and pelvic cavity with chocolate cysts in the bilateral ovaries and intraperitoneal rupture of the left ovarian cyst (blue arrow) and grade IV endometriosis. The rectosigmoid junction was anteriorly entrapped by dense fibrotic tissue (black arrow).

The upper rectum was anteriorly entrapped by dense fibrotic tissue which distorted and caused a stenosis of the lumen. The surgery included adhesiolysis, anterior resection with diverting end colostomy/distal mucous fistula and total abdominal hysterectomy with bilateral salpingo-oophrectomy. The post-operative period was uneventful. On the tenth post-operative day she was discharged on Danazol 200 mg twice daily for six months. Histopathological examination of the resected specimen revealed endometrial glands and stroma embedded in the submucosa, muscle, subserosal and serosal surface of the rectum and both the fallopian tubes and ovaries (Figure 3 ).

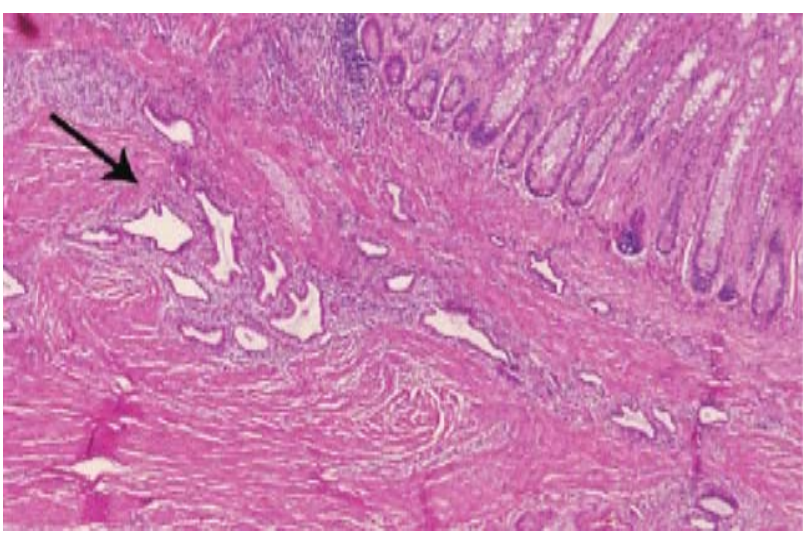

Figure 3: Hisptopatholgical examination of the resected specimen revealed endometriosis. The tissue composed of stroma and glands of endometrium (blackarrow). (HE X 100) 
On follow-up, she tolerated the drug well with minimal symptoms of pseudo-menopause. The colostomy was closed three months later. She remained stable during the one-year follow up. Her serial CA-125 remained within the normal limit.

\section{DISCUSSION}

Endometriosis is a common disease of unknown etiology. Extra-gonadal endometriosis is rarely diagnosed preoperatively. Presentations to general surgeons may be atypical and pose diagnostic difficulty by mimicking other acute diseases ${ }^{5}$.

The "neurologic hypothesis" and "infiltration or invasion phenomenon" is a new concept in the pathogenesis of endometriosis: lesions infiltrate the large bowel wall along the nerves, at a distance from the primary lesion. The mucosa is rarely involved as it is poorly innervated. Endometriosis infiltrating the muscularis propria may lead to localized fibrosis in the bowel wall, strictures, and bowel obstruction ${ }^{6}$.

The rectosigmoid is the most common site (70 \%) followed by the small bowel (1\%-7\%) for intestinal endometriosis. The true incidence of endometriosis causing bowel obstruction is unknown although complete obstruction of the bowel lumen occurs in less than $1 \%$ of cases $^{7}$.

The diagnosis of endometriosis may be suspected on the basis of the clinical history. Forty percent of the patients present symptoms in a cyclic manner, which are usually related with menses ${ }^{7}$.In our patient, symptoms relapsed irregularly and were not related with menses.

It is difficult to establish a preoperative diagnosis of $\mathrm{Gl}$ endometriosis, because Gl tract symptoms can mimic a wide spectrum of diseases. However, endometriosis of the large bowel should be suspected in young, nulliparous patients with abdominal pain, in conjunction with signs of obstruction ${ }^{8}$.

In our case also, as in others previously reported in the literature, ${ }^{3,4,8,9}$ it was impossible to establish an accurate preoperative diagnosis due to the vagueness of symptoms.

Although endoscopic diagnosis of colonic endometriosis has been reported, the mucosa is usually normal or shows minimal mucosal abnormalities and an extrinsic process or fibrosed stenoses ${ }^{10}$.

CT is not the primary imaging modality for evaluation of bowel endometriosis, although it can occasionally demonstrate a stenosing rectosigmoid mass. Multislice $\mathrm{CT}$, combined $\mathrm{MRI}$ and rectal endoscopic ultrasonography have recently been proposed to evaluate the depth of the rectal wall infiltration by endometriosis ${ }^{7}$.

Laparoscopy remains the only modality to confirm the presence of intestinal endometriosis prior to laparotomy and even treatment of intestinal endometriosis. Furthermore, recovery from laparoscopic surgery is better than from traditional laparotomy ${ }^{11}$.

There is great interest in the use of serum markers to diagnose endometriosis. Tumor marker CA-125 has been used to monitor the progress of endometriosis. For the accidental finding of intestinal endometriosis with absent or mild symptoms of obstruction, hormone therapy with danazol or $\mathrm{GnRH}$ analogs may be considered $^{11}$.

Surgical resection of the moderately to severely obstructed endometriotic bowel has been shown in a previous study to be safe and to provide long-term symptomatic relief. Perioperative frozen section biopsy is suggested in the case of inadequate resection to rule out carcinoma, which overall accounts for $1 \%$ of the cases $^{7,11}$.

More than $30 \%$ of cases will have disease recurrence after medical or surgical treatment. Postsurgical administration of danazol or $\mathrm{GnRH}$ analogs has lengthened the pain-free interval and decreased symptom recurrence rates in patients following surgery with severe endometriosis ${ }^{12}$.

In this paper, we report a rare presentation of endometriosis characterized by an acute large bowel obstruction. The report points out that endometriosis remains a challenging condition for clinicians, especially, as in our case, when the symptoms are not related to menses. Intestinal endometriosis should be considered in reproductive women with Gl symptoms and/or pelvic pain in conjunction with signs of intestinal obstruction or an intestinal mass of unknown origin. 


\section{REFERENCES}

1. Keane TE, Peel AL. Endometrioma:An intra-abdominal troublemaker. Dis Colon Rectum. 1990;33:963-5.

2. Lin YH, Kuo LJ, Chuang AY, Cheng TI, Hung CF. Extrapelvic endometriosis complicated with colonic obstruction. J Chin Med Assoc. 2006;69:47-50.

3. Paksoy M, Karabicak I, Ayan F, Aydogan F. Intestinal obstruction due to rectal endometriosis. Mt Sinai J Med. 2005;72:405-8.

4. Kim JS, Hur H, Min BS, Kim H, Sohn S, Cho C H, et al. Intestinal Endometriosis mimicking carcinoma of Rectum and Sigmoid Colon: A Report of Five Cases .J Yonsei Med. 2009;50(5):732-5.

5. Katsikogiannis N, Tsaroucha A, Dimakis K, Sivridis E, Simopoulos C. Rectal endometriosis causing colonic obstruction and concurrent endometriosis of the appendix: a case report. J Med Case Reports. 2011;5(1):320.

6. Anaf V, El Nakadi I, Simon P, Van de Stadt J, Fayt I, Simonart T, Noel JC. Preferential infiltration of large bowel endometriosis along the nerves of the colon. Hum Reprod. 2004;19:996-1002.
7. Cameron IC, Rogers S, Collins MC, Reed MW. Intestinal endometriosis: presentation, investigation, and surgical management. Int J Colorectal Dis. 1995;10:83-6.

8. Dimoulios P, Koutroubakis IE, Tzardi M, Antoniou P, Matalliotakis IM, Kouroumalis EA. A case of sigmoid endometriosis difficult to differentiate from colon cancer. BMC Gastroenterol. 2003;3:18.

9. Kaufman LC, Smyrk TC, Levy MJ, Enders FT, Oxentenko AS. Symptomatic intestinal endometriosis requiring surgical resection: clinical presentation and preoperative diagnosis. Am J Gastroenterol. 2011; 106(7):1325-32.

10. Averbach M, Abrao MS, Podgaec S, Correa P. Rectal endometriosis. Gastrointest Endosc. 2005;62:978-9.

11. Modesitt SC, Tortolero-Luna G, Robinson JB, Gershenson DM, Wolf JK. Ovarian and extraovarian endometriosis associated cancer. Obstet Gynecol. 2002;100:788-95.

12. Soysal S, Soysal ME, Ozer S, Gul N, Gezgin T. The effects of post-surgical administration of goserelin plus anastrozole compared to goserelin alone in patients with severe endometriosis: a prospective randomized trial. Hum Reprod. 2004;19:160-7. 